\{Reprinted with Permission\}

\title{
Joint Consensus Statement - Safety Precautions for Doing Echocardiography and Image Acquisition during COVID 19: Indian Academy of Echocardiography, Bangladesh Cardiac Society, Cardiac Society of Nepal, and Sri Lanka College of Cardiology
}

Rakesh Gupta ${ }^{1}$, S. K. Parashar ${ }^{2}$, H. K. Chopra ${ }^{3}$, Sameer Shrivastava ${ }^{4}$, Satish C. Govind ${ }^{5}$, Abdullah Al Shafi Majumder ${ }^{6}$, A. K. M. Monwarul Islam ${ }^{6}$, Mohsin Ahmed ${ }^{6}$, A. K. M. Mohibullah ${ }^{6}$, Yadav Kumar Deo Bhatt ${ }^{7}$, Deewakar Sharma ${ }^{8}$, Chandra Mani Adhikari ${ }^{9}$, Dipanker Prajapati ${ }^{9}$, M. R. Mubarak $^{10}$, Stanley Amarasekara ${ }^{11}$, G. Vijayraghavan ${ }^{12}$, P. Krisham Raju ${ }^{13}$, A. V. Anjaneyulu ${ }^{14}$, R. Alagesan $^{15}$, R. R. Kasliwal ${ }^{16}$, Rajan J. Manjuran ${ }^{17}$, S. Shanmugasundrum ${ }^{18}$, V. Amuthan ${ }^{19}$, S. K. Kaushik $^{20}$, S. T. Yavagal' ${ }^{21}$, Debika Chatterjee ${ }^{22}$, K. Raghu' ${ }^{13}$, C. K. Ponde ${ }^{23}$, Manish Bansal' ${ }^{16}$, G. Gnanavelu $^{24}$, Rahul Mehrotra ${ }^{25}$, Sunil Bohra ${ }^{26}$, S. Veermani ${ }^{27}$, U. P. Singh ${ }^{28}$, G. Rajesh ${ }^{29}$, K. M. Krishnamoorti ${ }^{30}$, Rishikesh Shah ${ }^{31}$

${ }^{1}$ JROP Institute of Echocardiography, Ultrasound and Vascular Doppler, Delhi, India, ${ }^{2}$ Metro Heart Institute, New Delhi, India, ${ }^{3}$ Moolchand Medicity, New Delhi, India, ${ }^{4}$ Fortis Escort's Heart Institute, New Delhi, India, ${ }^{5}$ Narayana Institute of Cardiac Sciences, Narayan Health City, Bengaluru, Karnataka, India, ${ }^{6}$ National Institute of Cardiovascular Diseases, Dhaka, Bangladesh, ${ }^{7}$ Norvic Hospital, Kathmandu, Nepal, 8HAMS Hospital, Kathmandu, Nepal, ${ }^{9}$ Shahid Gangalal National Heart Center, Kathmandu, Nepal,

${ }^{10}$ Lanka Hospital, Colombo, Sri Lanka, ${ }^{11}$ National Hospital of Sri Lanka, Colombo, Sri Lanka, ${ }^{12}$ Kerala Institute of Medical Sciences, Trivandrum, Kerala, India, ${ }^{13}$ Care Hospital, Hyderabad, Telangana, India,

${ }^{14}$ Focus Clinics Panjagutta, Hyderabad, Telangana, India, ${ }^{15} \mathrm{KM}$ Speciality Hospital, Chennai, Tamil

Nadu, India, ${ }^{16}$ Medanta Medicity, Gurgaon, Haryana, India, ${ }^{17}$ Pushpagiri Institute of Medical Sciences and Research Centre, Tiruvalla, Kerala, India, ${ }^{18}$ Billroth Hospital, Chennai, Tamil Nadu, India, ${ }^{19}$ Jeyalakshmi Heart Center, Madurai, Tamil Nadu, India, ${ }^{20} \mathrm{GBH}$ American Hospital, Udaipur, Rajasthan, India, ${ }^{21}$ Trinity Hospital and Heart Foundation, Bengaluru, Karnataka, India, ${ }^{22}$ RNT NH Institute of Cardiac Sciences, Kolkata, West Bengal, India, ${ }^{23}$ P. D. Hinduja National Hospital and MRC, Mumbai, Maharashtra, India, ${ }^{24}$ Ganesh Heart Care Center, Chennai, Tamil Nadu, India, ${ }^{25}$ MAX Superspeciality Hospital, New Delhi, India, ${ }^{26}$ Hosmat Hospital, Bengaluru, Karnataka, India, ${ }^{27}$ Madurai Medical College, Madurai, Tamil Nadu, India, ${ }^{28}$ Prime Diagnostic Centre and Heart Institute, Chandigarh, India, ${ }^{29}$ Medical

College, Calicut, Kerala, India, ${ }^{30}$ Sree Chitra Tirunal Institute for Medical Sciences and Technology,

Trivandrum, Kerala, India, ${ }^{31}$ Jupiter Hospital, Thane, Maharashtra, India

\begin{abstract}
Keywords:
COVID-19,

echocardiography,

An echocardiographic investigation is one of the key modalities of diagnosis in patients suffering from COVID 19, especially if they are elderly, have associated comorbid conditions, and pregnant. Hence, it is becoming extremely essential to look into the correct safety precautions, health-care image acquisition, joint statement, safety precautions professionals must take while conducting an echo investigation. At the same time, a focused echo examination aimed at correct imaging view acquisition in the shortest possible time is the need of the hour. The decision matrix formulated for conducting an echocardiographic evaluation is based on the presence or absence of cardiological comorbidity vis-a-vis positive or suspected for COVID19. The safety measures and image acquisition have been constructed keeping in mind the current safety precautions by the World Health Organization, the Centers for Disease Control and Prevention, and the Ministry of Health and Family Welfare, India.
\end{abstract}

Abstract

(Cardiovasc. j. 2020; 13(1): 97-101)

Address for correspondence: Dr. Rakesh Gupta, JROP Institute of Echocardiography, Ultrasound and Vascular Doppler, C 1/16, Ashok Vihar II, Delhi 110 052, India. E mail: rakeshecho@gmail.com

- 2020 Journal of the Indian Academy of Echocardiography \& Cardiovascular Imaging | Published by Wolters Kluwer Medknow 


\author{
Abbreviations \\ COVID-19 = Corona Virus Disease-19 \\ TTE $=$ Transthoracic echocardiography \\ $\mathrm{LV}=$ Left ventricle \\ $\mathrm{RV}=$ Right ventricle \\ $\mathrm{RA}=$ Right atrium \\ ICU = Intensive care unit \\ $\mathrm{OPD}=$ Out patient department \\ $\mathrm{PPE}=$ Personal protection Equipment \\ PLAX $=$ Parasternal long axis \\ $\mathrm{CFM}=$ Colour flow mapping \\ PSAX $=$ Parasternal short axis \\ $\mathrm{PW}=$ Pulse wave \\ RVOT $=$ Right ventricular outflow tract \\ $\mathrm{AP} 4 \mathrm{C}=$ Apical 4 chamber \\ MV = Mitral Valve \\ TAPSE = Tricuspid annular plane systolic \\ excursion \\ CCTDI $=$ Color coded tissue doppler imaging \\ AP2C = Apical 2 chamber \\ AP3C $=$ Apical 3 chamber \\ $\mathrm{AP} 5 \mathrm{C}=$ Apical 5 chamber \\ LVOT $=$ Left ventricular outflow tract \\ IVC $=$ Inferior vena cava \\ TEE $=$ Transesophageal echocardiography
}

\section{Introduction:}

The global coronavirus disease 2019 (COVID-19) pandemic is an unprecedented, rapidly spreading public health emergency which spread over 200 countries with $18,039,067$ cases with 689,100 deaths and 11,339,675 recovered patients as on August 02, 2020. Of the remaining $6,010,292$ cases, the major challenge is $65,776(1 \%)$, who are hospitalized in serious and critical conditions. ${ }^{1}$ Another major challenge is COVID-19 recovered patients $(11,339,675)$, who are coming back with their cardiovascular symptoms, requiring noninvasive evaluation including echocardiography.

The COVID 19 virus has affected the provision of health care services all over the world,${ }^{2}$ including South Asia. Health-care workers are at higher risk because this virus can spread very easily. This is especially true for the echocardiographers due to the close contact involved in the performance of echocardiographic studies. The virus carries a relatively high mortality and morbidity risk, particularly for certain populations (the elderly, the chronically ill, the immunocompromised, and, possibly, pregnant women). ${ }^{3}$ Given the risk of cardiovascular complications in the setting of COVID-19, including preexisting cardiac disease, acute cardiac injury, and drug-related myocardial damage ${ }^{4}$ echocardiography services are required in certain selected cases in the care of patients with suspected or confirmed COVID-19. Consequently, echocardiography providers will have a high risk of exposure to COVID-19. ${ }^{5}$ Despite the growing evidence of cardiovascular complications associated with the novel COVID19 , there is very little data regarding the performance of transthoracic echocardiography (TTE) in a step-wise method, especially views that are to be acquired in focused echocardiography for subsequent analysis.

The Indian Academy of Echocardiography along with the Bangladesh Cardiac Society, Cardiac Society of Nepal, and Sri Lanka College of Cardiology acknowledges the sincere efforts of all the various bodies (viz., your institute or hospital, local and state health authorities, Ministry of Health and Family Welfare, and Indian Council of Medical Research) in devising this paper. The purpose of this position paper is to supplement, not to override, valuable guidance from any of these bodies.

\section{Indications for transthoracic echocardiography}

TTE should be performed in patients who are COVID-19 suspected or confirmed only when the echocardiography study is expected to provide clinical benefit. Patients with elevated cardiac biomarkers are more likely to exhibit cardiac abnormalities in the form of reduced left ventricular function, dilated right ventricle (RV)-right atrium (RA), and reduced RV systolic function. In appropriately selected patients, TTE can guide COVID-19 cardiac involvement, complications, and clinical management. ${ }^{6}$

\section{Safety precautions}

The most part of safety precautions is that in today's scenario, many asymptomatic patients are visiting echocardiography labs for their cardiac evaluation. All patients should be treated like COVID-19 suspected and appropriate precautions as mentioned below should be taken. These safety precautions should be such that they apply to most of the developing countries in this part of the 
world. They should be innovative, cost effective, and less time consuming. Hence, considering these points, the following guidelines were made:

1. The echocardiography machine should be separate for COVID-19 ward/intensive care unit and COVID-19-suspected ward and outpatient department.

2. In all indoor patients of COVID-19, focused echocardiography should be done bedside with complete personal protection equipment.

3. The echocardiography machine should be properly covered with a disposable plastic sheet.

4. The probes should be cleaned thoroughly with soap and water solution or sanitizer/Cidex swab after each echocardiography examination.

5. The examination bed should be covered with a disposable plastic sheet that can be cleaned with sodium hypochlorite solution after each echocardiography examination.

6. Only trained cardiologists, preferably young without any comorbidities, should be deputed for doing echocardiography tests for the COVID-19-positive or -suspected patients. Elderly physicians with or without comorbid conditions should be discouraged to perform echocardiography by themselves.

7. To perform the echocardiography test: The cardiologist should be wearing a Level 3 personal protective equipment (PPE) gear while working in the COVID-19 ward, and at least Level 2 PPE gear, if working in the COVID-19-suspected ward.[7] The donning and doffing of PPE gears should be done as per guidelines. ${ }^{6}$

8. There should be a plastic curtain between the patient and the cardiologist doing an echocardiography examination.

9. There should be the provision of a heavy-duty exhaust fan near the patient's bed, away from the examiner.

10. Under no circumstances, the examiner should sit on the patient's bed for performing echocardiography.

11. The patient coming for the examination should wear a face mask and front-opening shirt/gown without any banyan/vest.
12. In this part of the world, the majority of echocardiography labs have a single room for performance and reading of echocardiography, leading to a high traffic area. All sanitization procedures should be adopted for sanitization of computer, door, handles, metallic parts, switchboard with cidex, and sodium hypochlorite solution. ${ }^{8-10}$

13. Various detailed precautions are advised in the guidelines published by the Cardiological Society of India ${ }^{7}$ and other cardiac societies.

\section{View acquisition}

A focused study should be performed quickly. The views, which are acquired in these circumstances without compromising the quality of an exam, should be to the point and useful for subsequent analysis, without any electrocardiogram gating. The number of views will be the minimum necessary for the purpose of making the decision to minimize the duration of an

examination. ${ }^{11,12}$

\section{Parasternal window}

a) Parasternal long-axis view with color flow mapping (CFM) covering mitral and aortic valve (.avi clip).

b) RV-RA inflow view with CFM (.avi clip).

c) Parasternal short-axis (PSAX) view at aorta with CFM at pulmonic valve and tricuspid valve (.avi clip).

d) PSAX view at aorta with CFM at pulmonic valve and pulse wave (PW) spectrum at right ventricular outflow tract (Doppler waveform).

\section{Apical window}

a) Apical four-chamber (AP4C) view with $\mathrm{CFM}$ on mitral and tricuspid valves (.avi clip).

b) $\mathrm{AP} 4 \mathrm{C}$ view with $\mathrm{CFM}$ at the mitral valve (MV) and PW Doppler spectrum pattern at MV (Doppler waveform).

c) AP4C view with color-coded tissue Doppler imaging (CCTDI) at the septum and PW Doppler spectrum pattern at mitral annulus (Doppler waveform).

d) $\mathrm{AP} 4 \mathrm{C}$ view with M-Mode at tricuspid annulus (Doppler waveform). It is also known as tricuspid annular plane systolic excursion. 
e) AP4C view with CCTDI at RV free wall and PW Doppler spectrum pattern at tricuspid annulus (Doppler waveform).

f) Apical two-chamber view with CFM at the MV (.avi clip).

g) Apical three-chamber view with CFM at mitral and aortic valves (.avi clip).

h) Apical five chamber (AP5C) view with CFM at mitral, aortic, and tricuspid valves (.avi clip).

i) $\mathrm{AP} 5 \mathrm{C}$ view with $\mathrm{CFM}$ at aortic valve with the PW spectrum at the left ventricular outflow tract (Doppler waveform).

j) Apical focused RV view with CFM at the tricuspid valve (.avi clip).

k) Apical focused RV view with CFM at the tricuspid valve and CW Doppler spectrum pattern across the tricuspid valve (Doppler waveform).

\section{Subcostal window}

a) Subcostal view with CFM at the tricuspid view (.avi clip)

b) Inferior vena cava dynamic view (.avi clip).

\section{Stress echocardiography}

The treadmill or bicycle stress echocardiography tests in patients with COVID-19 may lead to exposure because of deep breathing and/or coughing during exercise. These tests should generally be deferred or converted to pharmacologic stress echocardiography, if essential, for patient management.

\section{Transesophageal echocardiography}

Transoesophageal echocardiography (TEE) carries a very high risk of exposure to aerosolized secretion and subsequently carries an increased risk of COVID-19 transmission. In addition, the symptom profile of COVID 19 is imprecise and heterogeneous, making it hard to assess who is currently a vector. Moreover, it is presumed that COVID-19 is now widespread in this region. We, therefore, recommend the following: ${ }^{13-15}$

a) All routine TEE cases should be canceled. Alternative imaging modalities may be considered that may provide the necessary information. b) Only those cases, where it is still felt that a TEE will have a significant and immediate impact on patient management, should be undertaken in consultation with other involved specialties.

c) Surgical colleagues should be involved, ensuring that the operation remains possible if indicated, or with microbiology colleagues to discuss potential treatment strategies for the management of infective endocarditis.

d) Perioperative TEE should be performed only if there is a favorable risk: benefit ratio while taking into account the added risk of COVID19 transmission. The added airway protection provided by a cuffed endotracheal tube and closed circuit ought to reduce the risk of aerosol generation in an anesthetized, paralyzed, and ventilated patient. However, the longer duration and exposure to airway secretions may increase infection risk. Care must be taken to avoid accidental extubation and disconnection of the anesthetic circuit. ${ }^{16-}$ 18

e) Appropriate PPE should be worn by all personnel involved in all TEE examinations, including the use of an appropriately fit tested mask.

f) Appropriate decontamination of the procedure room and equipment should be undertaken after each procedure.

\section{Summary}

The present COVID-19 pandemic is a medical emergency of an unprecedented scale in recent human history with constantly evolving strategies. As we continue to handle the onslaught of this pandemic, strategies may continue to evolve further. In any given patient with COVID-19, ${ }^{19-21}$ clinical assessment along with inflammatory markers will be necessary to determine whether the patient is experiencing a primary coronary event or other cardiac involvement. If there is a suspicion of cardiac involvement, the risk to benefit ratio of performing echocardiography becomes extremely helpful in deciding the need for the same. If the echocardiography is performed, it should be focused and should be performed following all the protocols as outlined above, especially the health-care provider's protection. 
The stress echocardiography should be deferred, and, if at all it is a necessity, then pharmacological echocardiography should be done. Similarly, the performance of TEE should be considered only in highly selected cases that are mandatory for a patient undergoing emergency interventional procedures. Other imaging modalities should be considered in this place to proving the required necessary information.

\section{Conflict of Interest - None.}

\section{References:}

1. Available from: https://www.worldometers.info/ coronavirus/. [Last accessed on 2020 Aug 01].

2. WHO Director General's Opening Remarks at the Media Briefing on COVID-19. 11 March 2020. Available from: https://www.who.int/dg/speeches/detail/who-directorgeneral-s-opening-remarks-at-the-mediabriefing on covid 1911 march 2020. [Last accessed on $2020 \mathrm{Mar}$ 19, and Published 2020].

3. Zhou F, Yu T, Du R, Fan G, Liu Y, Liu Z, et al. Clinical course and risk factors for mortality of adult inpatients with COVID-19 in Wuhan, China: A retrospective cohort study [published correction appears in Lancet. 2020 Mar 28;395(10229):1038] [published correction appears in Lancet. 2020 Mar 28;395(10229):1038]. Lancet 2020; 395: 1054-1062.

4. Zheng YY, Ma YT, Zhang JY, Xie X. COVID-19, and the cardiovascular system. Nat Rev Cardiol 2020;17: 259-260.

5. Kirkpatrick JN, Mitchell C, Taub C, Kort S, Hung J, Swaminathan M. ASE Statement on Protection of Patients and Echocardiography Service Providers During the 2019 Novel Coronavirus Outbreak: Endorsed by the American College of Cardiology. $J$ Am Soc Echocardiogr 2020; 33: 648-653.

6. BSE Clinical Guidance Regarding the Provision of Echocardiography during the COVID-19 Pandemic, updated Guidance on a Provision of Echocardiography. 02.04.2020.

7. Gupta R, Das MK, Mohanan PP, Deb PK, Parashar SK, Chopra HK, et al. Cardiological Society of India document on safety measure during echocardiography evaluation of cardiovascular disease in the time of COVID-19. Indian Heart $J$ 2020; 73:145-150.

8. Picard MH, Weiner RB. Echocardiography in the Time of COVID-19. J Am Soc Echocardiogr 2020; 33: 674675 .

9. Dweck MR, Bularga A, Hahn RT, Bing R, Lee K K, Chapman A R, et al. Global evaluation of echocardiography in patients with COVID-19 [published online ahead of print, 2020 Jun 18]. Eur Heart $J$ Cardiovasc Imaging 2020; jeaa178.
10. Sattarzadeh BR, Almassi N, Moshkani Farahani M, Movassaghi N, Khosravi A, Mirdamadi A, et al. The Iranian Society of Echocardiography (ISE) statement on performing echocardiography during the COVID19 pandemic. Curr Probl Cardiol 2020; 45: 100620.

11. Cameli M, Pastore MC, Soliman Aboumarie H, Mandoli GE, D'Ascenzi F, Cameli P, et al. Usefulness of echocardiography to detect cardiac involvement in COVID-19 patients [published online ahead of print, 2020 Jul 12]. Echocardiography 2020;10.1111/ echo. 14779 .

12. Ward RP, Lee L, Ward TJ, Lang RM. Utilization and appropriateness of transthoracic echocardiography in response to the COVID-19 pandemic. J Am Soc Echocardiogr 2020; 33: 690-691.

13. Gackowski A, Lipczyñska M, Lipiec P, Szymañski P. Echocardiography during the coronavirus disease 2019 (COVID-19) pandemic: Expert opinion of the Working Group on Echocardiography of the Polish Cardiac Society. Kardiol Pol 2020; 78: 357-363.

14. Bracco D. Safe (r) transesophageal echocardiography and COVID-19. Can J Anaesth 2020; 67: 1101-1103.

15. Skulstad H, Cosyns B, Popescu BA, Galderisi M, Di Salvo G, Donal E, et al. COVID-19 pandemic and cardiac imaging: EACVI recommendations on precautions, indications, prioritization, and protection for patients and healthcare personnel. Eur Heart $J$ Cardiovasc Imaging 2020; 21: 592-598.

16. Flower L, Olusanya O, Madhivathanan PR. The use of point-of-care lung ultrasound and echocardiography in the management of coronavirus disease 2019 (COVID19) [published online ahead of print, 2020 May 15]. J Cardiothorac Vasc Anesth 2020; S1053- 0770 (20) 3043030434 .

17. Jain A. Preventing contamination during transesophageal echocardiography in the face of the COVID-19 pandemic [published online ahead of print, 2020 Apr 13]. J Cardiothorac Vasc Anesth 2020; S10530770(20)30369-4.

18. Lee TW, Enns J. Making transesophageal echocardiography safer during COVID-19: Is there a role for probe protective equipment (pPE)? [published online ahead of print, 2020 May 18]. Can J Anaesth 2020;1-2.

19. Harikrishnan S, Mohanan PP, Chopra VK, Ambuj R, Sanjay G, Bansal M, et al. Cardiological society of India position statement on COVID-19 and heart failure. Indian Heart $J$ 2020; 72: 75-81.

20. Cameli M, Pastore MC, Henein M, Aboumarie HS, Mandoli GE, D'Ascenzi F, et al. Safe performance of echocardiography during the COVID-19 pandemic: A practical guide. Rev Cardiovasc Med 2020; 21: 217-223.

21. Wahi S, Thomas L, Stanton T, Taylor A, Mahadevan D, Evans G, et al. CSANZ imaging council position statement on echocardiography services during the COVID-19 pandemic. Heart Lung Circ 2020; 29: e78-83. 